https://helda.helsinki.fi

\title{
The effects of ovarian biopsy and blood sampling methods on salivary cortisol and behaviour in sows
}

\section{Yun, Jinhyeon}

2017

Yun , J , Björkman , S , Pöytäkangas , M \& Peltoniemi , O 2017 , ' The effects of ovarian biopsy and blood sampling methods on salivary cortisol and behaviour in sows ' , Research in Veterinary Science, vol. 114 , pp. 80-85 . https://doi.org/10.1016/j.rvsc.2017.03.004

http://hdl.handle.net/10138/307914

https://doi.org/10.1016/j.rvsc.2017.03.004

cc_by_nc_nd

acceptedVersion

Downloaded from Helda, University of Helsinki institutional repository.

This is an electronic reprint of the original article.

This reprint may differ from the original in pagination and typographic detail.

Please cite the original version. 
The effects of ovarian biopsy and blood sampling methods on salivary cortisol and behaviour in

sows

$4{ }^{A}$ Research Centre for Animal Welfare, Department of Production Animal Medicine, Faculty of

5 Veterinary Medicine, University of Helsinki, P.O.Box 57, 00014 Helsinki, Finland

$6 \quad$ B Production Animal Hospital, Department of Production Animal Medicine, Faculty of Veterinary

7 Medicine, University of Helsinki, Paroninkuja 20, 04920 Saarentaus, Finland

$8 \quad{ }^{\mathrm{C}}$ Corresponding author. Email: jinhyeon.yun@ helsinki.fi

Abstract

11 In reproductive physiology research, experimental animals are often subjected to stressful

12 procedures, including blood sampling and biopsy. In this present study, presence of pain or distress

13 induced by four different procedures was examined using a measurement of salivary cortisol levels

14 and activity observations in sows. The treatments were: 1) PAL: The ovary was palpated through

15 the rectum without snaring, 2) TUB: transvaginal ultrasound-guided biopsy of the ovary was

16 conducted without snaring, 3) SNA: a soft rope snare was placed around the maxilla, 4) CAT: A

17 soft rope snare was placed around the maxilla, and an intravenous catheter was inserted through the

18 ear vein of the sows. Activities, social cohesion and other pain-related behaviour, and salivary

19 cortisol concentrations were recorded. Salivary cortisol concentrations in CAT sows increased in

20 response to the procedure $(P<0.05)$, whereas the other treatments did not trigger a significant

21 response. The CAT sows had higher cortisol concentrations than the other groups for 10 min after

22 initiation of the procedures $(P<0.01)$, and they maintained higher cortisol levels than the PAL and

23 TUB groups 15 min post-treatment $(P<0.05)$. Furthermore, the CAT sows showed the highest 
24 frequency of head shaking $(P<0.001)$ and trembling behaviour $(P<0.05)$ during the $1 \mathrm{~h}$ post-

25 treatment. Summarizing, the catheterization procedure might induce a short-term pain or stress

26 response during and after the procedure in terms of pain-related behaviour and salivary cortisol

27 status. We suggest that TUB might not cause appreciable pain or distress.

28 Additional keywords: pain behaviour; stress response; glucocorticoid; luteal tissue; catheterization

1. Introduction

32 Björkman et al. (2016) examined the use of a transvaginal ultrasound-guided biopsy technique 33 (TUB) for the observation of the porcine corpus luteum together with its effects on reproductive 34 performance. In the past, a biopsy of the corpus luteum in pigs could be completed only through 35 anaesthesia and laparotomy (Ribeiro et al., 2007) or euthanasia (Conley and Ford, 1989), with 36 attendant sampling limitations and welfare concerns. The TUB was designed to provide an 37 alternative method, and possibly promote research gains and pig welfare. Consequently, Björkman 38 et al. (2016) suggested that this could be extensively utilized for longitudinal studies to investigate 39 corpus luteum mechanisms in pigs. However, it is not known whether a less invasive biopsy method 40 still causes distress to the pig.

41 A nonsurgical catheterization procedure through the auricular vein is well accepted and used in 42 numerous pig studies to collect frequent and sequential blood samples, minimizing the number of 43 times needles are inserted, thereby avoiding vascular injury and pain and distress. The method has 44 been verified as suitable for collecting blood samples through various studies also conducted in our 45 research stations (Peacock, 1991; Peltoniemi et al., 1995; Tast et al., 2001; Virolainen, 2005; Yun et 46 al., 2013). To date, however, the potential of the catheterization method to trigger a stress response, 
47 particularly in situations when pigs are tethered during the procedure and when their necks and ears

48 are bandaged during the sampling periods, has not been examined.

49 Measuring cortisol concentrations could be an effective method to assess pain and distress in

50 animals, particularly if they are physically restrained for research purposes (Weary et al., 2006;

51 Merlot et al., 2011). In lieu of a blood sample in which the stressful sampling methods could

52 significantly impact the biological process under investigation, measuring cortisol concentrations in

53 saliva represent a growing trend in welfare studies, and have been validated in numerous pig studies

54 (Geverink et al., 1999; Hillmann et al., 2008; Cook et al., 2013). Because the saliva sampling

55 method is non-invasive and less stressful compared with blood sampling, it might be possible to

56 collect multiple cortisol samples for measuring distress in pigs (Cook et al., 2013). In addition, it is

57 widely known that observations of pain-related behaviours and reduction in particular behaviours,

58 such as normal activity, are also useful for pain assessment in pig research (e.g. Weary et al., 2006).

59 Many benefits accrue from using the TUB or catheterization methods, particularly for taking

60 multiple samples and improving the welfare of experimental animals. However, it has not yet been

61 determined if the procedures are accompanied by pain and stress in the animal. We hypothesized

62 that both the TUB and the catheterization method would cause a similar short-term stress response

63 in the experimental sows, considering the degree of invasiveness of the procedures involved. In this

64 study, therefore, the short-term stress response of sows to TUB and catheterization was examined

65 through changes in physiological and behavioural variables.

66

67

2. Materials and methods

68 The study procedure was reviewed and approved by the Animal Experiment Board (ELLA) in

69 Finland, permission ESAVI/3331/04.10.03/2011. The experiment was conducted on a commercial

70 pig farm registered as an experimental research station in Vihti, southern Finland during 2015. 
72 Sows were housed in groups, approximately twenty per group pen $(20 \times 5 \mathrm{~m})$, where they were

73 allowed ad libitum access to water from a nipple drinker, and were fed a standard pregnancy diet

74 twice a day (08:00/16:00) via an automatic liquid feeding system. One day prior to the procedures,

75 all the sows included in the experiment were randomly paired, and each pair was separately housed

76 in pens $(2.5 \times 2.5 \mathrm{~m})$. Both group and individual pens consisted of a solid concrete floor with

77 abundant straw as bedding material. Before the procedures, each sow was individually transported

78 to the operation stall located in the corridor outside the room. The operation stall comprised a

79 conventional steel crate, a feeding trough and a rubber mat on the floor. After the treatments, the

80 sows were returned to the individual pen, staying with their original pen mates, and their behaviour

81 was monitored for one hour thereafter. The sow transportation both ways, between the pen and the

82 operation stall, was done gently within one minute, by a trained staff member.

84 A total of 32 weaned sows (Finnish Yorkshire $\times$ Large White; parity $3.9 \pm 0.6$ ) were randomly

85 selected for the experiment, approximately four days after weaning. The sows were assigned to four treatments: 1) Ovarian palpation (PAL; $\mathrm{N}=8)$ : The ovary of the sows was palpated through the rectum without snaring, 2) Biopsy (TUB; $N=8$ ): TUB of the ovary of the sows was conducted without snaring, 3) Snaring (SNA; $N=8$ ): A soft rope snare was placed around the maxilla of the sows, 4) Catheterization (CAT; $N=8$ ): A soft rope snare was placed around the maxilla, and an intravenous catheter was inserted through the ear vein of the sows. Sows in the PAL and SNA

91 groups were designed as control groups of the TUB and CAT sows, respectively. This experimental 92 setup was used to evaluate the separated stress factor induced by the TUB and catheterization per 93 se, while standardising other stressors, i.e., palpating and snaring. The sows of the PAL and TUB 94 groups were treated on the same day, and the sows of the SNA and CAT groups were treated on the 95 following day. All procedures, including the TUB and catheterization, lasted for approximately 10 
96 min per sow and they were performed at the prescribed time between 1200 and $1500 \mathrm{~h}$. The timing

97 and duration of the procedure according to the treatments were considered to standardize cortisol

98 circadian patterns on the proceeding day. One sow from TUB had to be excluded from data analysis

99 as we failed to collect a biopsy from the sow.

101 After sows were transported to the operation stall, faeces were removed from the rectum, and the 102 vulva was washed three times with a Povidone-Iodine solution (7.5\% Betadine, Leiras Oy, Helsinki, 103 Finland). The ovary of the sows in the PAL group was palpated through the rectum for 104 approximately $10 \mathrm{~min}$. The sows in both PAL and TUB groups were not snared during the process. 105 The TUB procedure was thoroughly described by Björkman et al. (2016). Briefly, the Tru-Cut 106 biopsy needle (16-gauge diameter, Zamar, Ultimate, PMO16-25, Poreč, Croatia), consisting of an 107 inner needle with a $1 \mathrm{~cm}$ specimen notch and an outer cutting cannula, was inserted into a needle 108 guide (Length $18 \mathrm{~cm}$; DBSE12X Biopsy kit, Esaote SpA, Maastricht, The Netherlands), which was 109 placed onto a $6.8 \mathrm{MHz}$ micro-convex array probe (Length $30 \mathrm{~cm}$; Endocavity probe, SE3123, 110 Esaote SpA, Maastricht, The Netherlands). The probe was connected to an ultrasound device 111 (MyLabOne Vet, Esaote SpA, Maastricht, The Netherlands), and placed into the vagina towards the 112 uterine cervix. Simultaneously, the ovary was palpated through the rectum and relocated towards 113 the caudal end of the cervix and the ultrasound probe. After the ovary appeared on the ultrasound 114 screen, the biopsy needle was placed through the vaginal wall and the ovarian surface into the 115 ovary. When in place, the inner needle was pushed about $1 \mathrm{~cm}$ into the ovarian tissue, followed by 116 the outer cannula, which cut and trapped ovarian tissue inside the notch.

117 2.2.2. Snaring (SNA) and catheterization through the auricular vein (CAT)

118 The sows in CAT and SNA were caught with a soft rope snare placed around the maxilla to 119 provide restraint for $10 \mathrm{~min}$ while being catheterized or just snared, respectively. The dorsal surface 
121 P, Berner Oy, Helsinki, Finland). The catheterization method was detailed previously (Virolainen,

122 2005; Yun et al., 2013). A tourniquet was tied around the base of the ear and a 13-gauge

123 intravenous catheter (Intraflon2, Vygon, Ecouen, France) was inserted into the auricular vein, and a

$12450 \mathrm{~cm}$ long vinyl tube (OD/ID of $1.5 \times 1.0 \mathrm{~mm}$, Steri-products, Australia) was threaded through the

125 catheter into the jugular vein. The 13-gauge catheter was removed, and an 18-gauge blunted needle

126 hub was inserted into the end of the vinyl tubing. A stopper was then inserted into the needle hub to

127 prevent blood backflow. The end of tube with the stopper was stored in a Velcro pouch attached to

128 the nape of the sow's neck. The neck together with the folded-ear was bound twice with a bandage.

129

130

131

132

133

134

135

136

137

138

139

140

\subsection{Sample collection and assays}

Five saliva samples from each sow were collected on synthetic swabs (Salivette ${ }^{\circledR}$ Cortisol, Sarstedt, Nümbrecht, Germany). The swabs were fixed with forceps and placed around the back teeth for approximately one minute by a trained researcher. If necessary, the researcher induced the sow to chew the swab by gently rubbing. In cases where sows were not able to move their jaws due to snaring, the researcher wiped around the back teeth with the swab to obtain the sample. The first and the last saliva samples from the sow were collected in the individual pens, and the other samples were collected at the operation stalls within over five minute intervals (Table 1). All saliva samples were collected in ice-chilled tubes, and centrifuged for $10 \mathrm{~min}$ at $1000 \times \mathrm{g}$. The samples were stored at $-20^{\circ} \mathrm{C}$ for subsequent analysis of cortisol.

Table 1. Scheme for saliva sampling from sows during palpation (PAL), biopsy (TUB), snaring (SNA) and catheterization (CAT).

\begin{tabular}{ccccc}
\hline & PAL & TUB & SNA & CAT \\
Time & $(\mathrm{n}=8)$ & $(\mathrm{n}=7)$ & $(\mathrm{n}=8)$ & $(\mathrm{n}=8)$ \\
\hline-5 & & Sampling at the individual pen & \\
\hline & & Sows are transported to the operation stall \\
\hline
\end{tabular}




\begin{tabular}{|c|c|c|c|c|}
\hline 0 & \multicolumn{4}{|c|}{ Sampling at the operation stall } \\
\hline & \multicolumn{4}{|c|}{ Procedures initiate according to the treatments } \\
\hline 5 & \multicolumn{4}{|c|}{ Sampling 5 min after the initiation of procedures } \\
\hline 10 & $\begin{array}{l}\text { Sampling } 10 \mathrm{~min} \\
\text { after the initiation } \\
\text { of the procedure }\end{array}$ & $\begin{array}{l}\text { Sampling } \\
\text { immediately after } \\
\text { collecting biopsy }\end{array}$ & $\begin{array}{l}\text { Sampling } \\
\text { immediately after } \\
\text { releasing a snare }\end{array}$ & $\begin{array}{l}\text { Sampling } \\
\text { immediately after } \\
\text { releasing a snare }\end{array}$ \\
\hline \multicolumn{5}{|c|}{ Sows are transported to the individual pen } \\
\hline 15 & & Sampling at $\mathrm{t}$ & individual pen & \\
\hline
\end{tabular}

142 Concentrations of salivary cortisol were analyzed in duplicate with a commercial

143 radioimmunoassay kit (ImmuChem ${ }^{\mathrm{TM}} \mathrm{CT}$ cortisol kit, MP Biomedicals, Orangeburg, NY, USA)

144 using a modified RIA method for saliva. Briefly, a calibration curve (from 0.5 to $50 \mathrm{ng} / \mathrm{ml}$ ) suitable

145 for saliva samples was made by diluting the highest calibrator of the kit with phosphate-buffered

146 saline (pH 7.5). $200 \mu 1$ of each saliva sample or diluted calibrator was added to antibody coated

147 tubes and incubated with $1 \mathrm{ml}$ of $125 \mathrm{I}$-labelled cortisol solution at $37{ }^{\circ} \mathrm{C}$ for $45 \mathrm{~min}$. The tubes were

148 decanted and counted in a gamma counter. Parallelism between undiluted and 4-fold diluted saliva

149 samples was $99 \%$. The quantification limit of the cortisol assay was $0.5 \mathrm{ng} / \mathrm{ml}$. The intra- and inter-

150 assay coefficients of variation were $6.5-9.7 \%$ and $9.7-12.1 \%$, respectively.

$151 \quad$ 2.4. Behavioural observations

152 All sows were video-recorded continuously for one hour after the procedures to monitor activities, 153 social cohesion and other pain-related behaviour. Internet protocol (IP) cameras (Niceview

154 NICECAM420WL, Niceview Corp.) were mounted in each pen. The sequence output was recorded 155 using IP-camera software (Blue Iris v.2.64, Perspective Software Corp.). The display resolution was $156640 \times 480$ pixels, and the frame rate was 2 FPS.

157 The CowLog v.2.0 (Hänninen and Pastell, 2009) behavioural recording program with a trained 158 observer was used for data analyses. The durations of activities were monitored, including standing 

recumbence: the sow lies on the sternum with udder concealed, (4) Lateral recumbence: the sow lies

162 on the lateral with udder exposed and head, hip bone and shoulder touches the ground. Furthermore, 163 the duration of social cohesion was assessed based on the distance between two sows in a pen.

164 When the distance between the heads of two sows in the individual pen was approximately greater 165 than sow mean body length, it was termed 'isolation'. In addition, 'desynchronization' was defined 166 as when sows showed different activities or poses against their pairs in the pen. Four parameters 167 selected from the literature (Hay et al., 2003; Noonan et al., 1994) were used to evaluate specific 168 normal (i.e., sniffing) or pain-related (i.e., rubbing, trembling and head shaking) behaviour in sows 169 after treatment: (1) Sniffing: The snout close to the floor with the head down, (2) Rubbing: Rubbing 170 or scratching the body against the pen walls, (3) Trembling: Shivering as if cold, (4) Head shaking:

171 Shaking head rapidly from side to side. These behaviours were represented by their occurrences 172 during one hour after treatment.

174 Statistical processing of all data was done in SAS v.9.4 (SAS Institute Inc., NC, USA, 2012).

175 Significant differences between treatment means were determined by LSD application, and set at $P$ $176<0.05$, and tendencies were determined if $P>0.05$ and $P<0.10$. An individual animal represented 177 an experimental unit.

178 A mixed model, using treatment as a fixed effect and pair as a random effect, was fitted to the data 179 for analysis of all the behaviour observations and cortisol concentrations according to the 180 treatments. We used multiple comparison procedures to analyse all the data, since the experimental 181 procedure was the sole fixed variable whilst housing, transportation and confining in the operation 182 stall were standardized between the treatments. Thereafter, post-hoc analyses using the Kenward183 Rogers procedure were performed to compare cortisol concentrations after treatment for CAT vs. 
184 PAL and TUB, and SNA vs. CAT, PAL and TUB sow groups, where significant differences were 185 found. Repeated measures with an 'unstructured' model were used to evaluate cortisol 186 concentrations before and after treatment.

187 Spearman rank correlation $\left(r_{s}\right)$ coefficients were used to examine interactions between the salivary 188 cortisol concentrations and behavioural observations in post-treatment sows. Pearson correlation $(r)$ 189 was applied to determine the parity effect on the salivary cortisol concentrations of the sow.

3. Results

\subsection{Saliva cortisol concentrations}

193 The average cortisol concentrations for all sows were $3.0 \pm 0.3,3.2 \pm 0.3,3.8 \pm 0.3,3.9 \pm 0.3$ and 194 $4.4 \pm 0.5(\mathrm{LS}$ mean $\pm \mathrm{SEM}) \mathrm{ng} / \mathrm{ml}$, according to the timing of treatments, i.e. $-5,0,5,10$ and $15 \mathrm{~min}$ from the initiation of treatment, respectively, and negatively correlated with parity of the sow ( $r=-$ $0.27, P<0.001)$.

197 The catheterization brought about an increase in saliva cortisol concentrations of the sows $(P<$ 198 0.05, Figure 1), while the cortisol levels associated with the other methods were not significantly 199 changed during the procedures $(P>0.10$, Figure 1$)$. The cortisol concentrations in CAT sows were 200 significantly greater than in sows in the other treatments for $10 \mathrm{~min}$ after initiation of the procedures 201 ( $P<0.01$, Table 2). Post-hoc comparisons showed that after CAT sows were returned to the 202 individual pens post-treatment, they still had higher saliva cortisol concentrations than non-snared 203 sows, i.e., PAL and TUB ( $P<0.05$, for both). However, saliva cortisol concentrations from the 204 sows in the SNA group were not significantly different to those of the PAL, TUB, or CAT sow 205 groups after treatment $(P>0.10)$. Sows in the TUB group tended to have higher cortisol 206 concentrations than those in the other groups before the experimental treatments, but the 


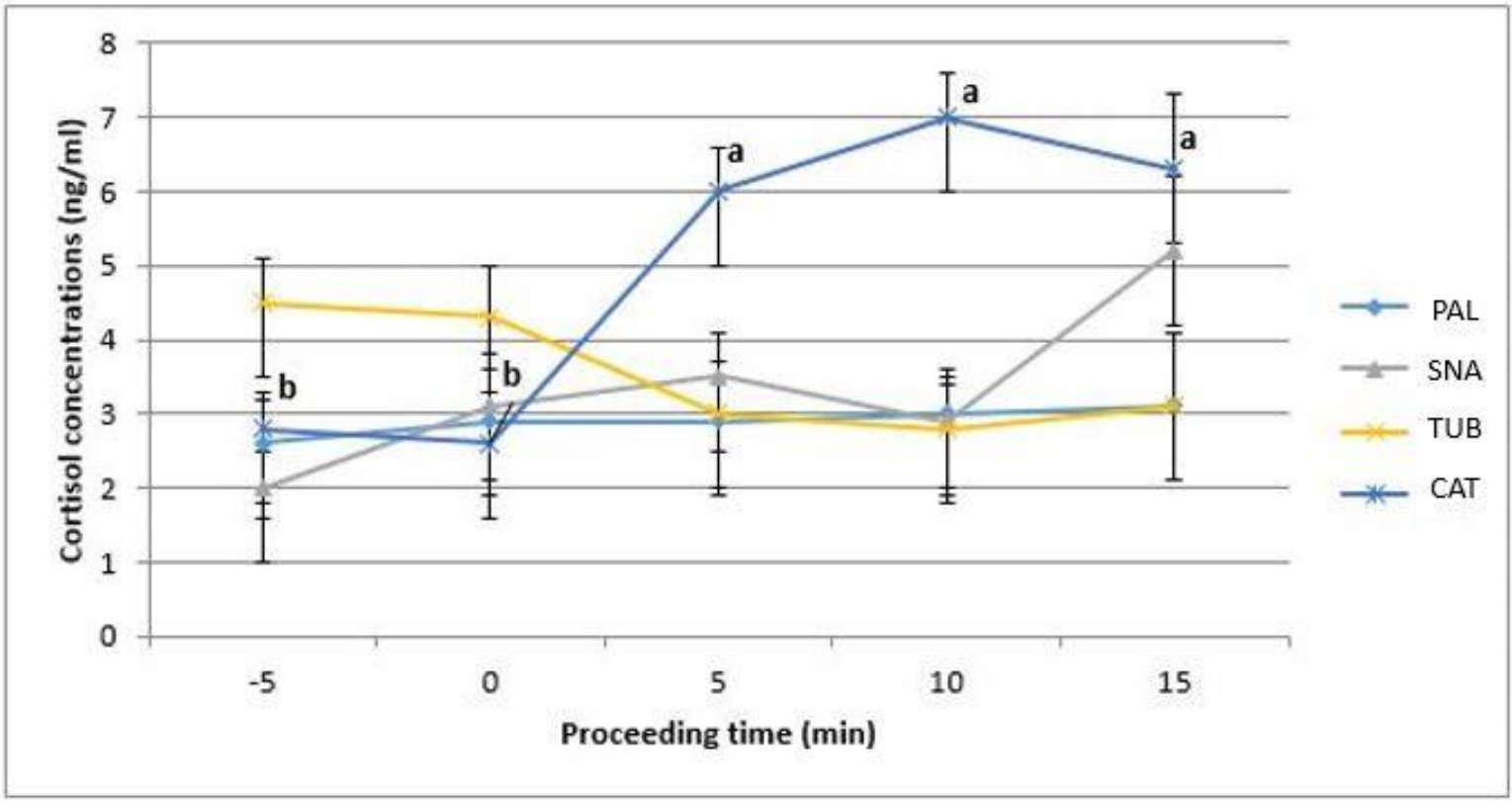

Figure 1. LS means and SE of sow saliva cortisol concentrations according to the experimental to demonstrate short term changes during the proceeding periods (total $n=31)$. Different letters $(a, b)$ indicate that the cortisol levels in the CAT treatment were significantly different according to the proceeding time $(P<0.05)$.

Table 2. Salivary cortisol concentrations in sows submitted to four different treatments: palpation (PAL), biopsy (TUB), snaring (SNA) and catheterization (CAT) ${ }^{\mathbf{1}}$.

\begin{tabular}{cccccc}
\hline & PAL & TUB & SNA & CAT & \\
$\mathrm{n}$ & 8 & 7 & 8 & 8 & $P$ value \\
\hline \multicolumn{2}{c}{ Cortisol concentration $(\mathrm{ng} / \mathrm{ml})$} & & & & \\
$-5,0 \mathrm{~min}^{2}$ & $2.7(0.5)$ & $4.4(0.6)$ & $2.6(0.5)$ & $2.7(0.5)$ & 0.09 \\
$5,10 \min ^{3}$ & $2.9(0.6)^{\mathrm{b}}$ & $2.9(0.6)^{\mathrm{b}}$ & $3.2(0.6)^{\mathrm{b}}$ & $6.5(0.6)^{\mathrm{a}}$ & $<0.001$ \\
$15 \min ^{\mathrm{b}}$ & $3.1(1.0)$ & $3.1(1.0)$ & $5.2(1.0)$ & $6.3(1.0)$ & 0.07
\end{tabular}

$219{ }^{1}$ All treatments proceeded between 0 and 10 min per sow.

$220 \quad{ }^{2}$ Repeated measures carried out LS Mean (SE) from -5 to 0 min according to the sampling scheme. 
$221{ }^{3}$ Repeated measures carried out LS Mean (SE) from 5 to 10 min according to the sampling scheme.

$222{ }^{\mathrm{a}, \mathrm{b}}$ Different superscript letters indicate that there were significant differences between variables $(P$ $223<0.001)$.

224

226 The SNA sows tended to sit for longer in the pen, and had longer desynchronized action against 227 their pen mates than the sows in other treatments $(P=0.09$ and $P<0.05$, respectively, Table 3$)$. The 228 TUB sows tended to spend longer time for lateral laying down that the other sows $(P=0.09$, Table $2293)$

230 During the $1 \mathrm{~h}$ post-treatment, sows in PAL showed a higher frequency of sniffing behaviour than 231 sows in SNA or CAT $(P<0.01$, Table 3$)$. The frequency of sniffing was a negatively correlated 232 with saliva cortisol in the post-treatment sows $\left(r_{s}=-0.28, P<0.01\right.$, Table 3$)$.

233 The highest frequency of head shaking was recorded for the CAT sows followed, respectively, by 234 SNA and PAL sows $(P<0.001$, Table 3$)$, and TUB sows did not differ from SNA or PAL sows $(P$ $235>0.10$ ). The CAT sows also showed higher frequency of trembling behaviour than the other treated 236 sows $(P<0.05$, Table 3$)$. The frequencies of head shaking, trembling, and rubbing behaviour were 237 correlated with saliva cortisol concentrations in posttreatment sows $\left(r_{s}=0.36, P<0.001 ; r_{s}=0.35\right.$, $238 P<0.001 ; r_{s}=0.22, P<0.05$, respectively).

239 Table 3. Effects of palpation (PAL), biopsy (TUB), snaring (SNA) and catheterization (CAT) on 240 activities, social cohesion and other specific normal or pain-related behaviour during $1 \mathrm{~h}$ after the 241 procedures in sows $(n=31){ }^{1}$

\begin{tabular}{cccccc}
\hline & PAL & TUB & SNA & CAT & \\
$\mathrm{n}$ & 8 & 7 & 8 & 8 & $P$ value \\
\hline
\end{tabular}

Activities, sec / $1 \mathrm{~h}$

$\begin{array}{llllll}\text { Standing/walking } & 828.8(257.0) & 583.4(274.8) & 1011.0(257.0) & 677.0(257.0) & 0.68\end{array}$ 


\begin{tabular}{|c|c|c|c|c|c|}
\hline Sitting & $29.9(14.5)$ & $19.1(15.5)$ & $62.0(14.5)$ & $10.6(14.5)$ & 0.09 \\
\hline $\begin{array}{l}\text { Sternal } \\
\text { recumbence }\end{array}$ & $2363.1(286.4)$ & $1634.9(306.1)$ & $1789.1(286.4)$ & $2051.1(286.4)$ & 0.33 \\
\hline $\begin{array}{l}\text { Lateral } \\
\text { recumbence }\end{array}$ & $296.1(315.8)$ & $1391.2(333.3)$ & $737.9(315.8)$ & $833.6(315.8)$ & 0.07 \\
\hline \multicolumn{6}{|c|}{ Social cohesion, sec / $1 \mathrm{~h}$} \\
\hline Isolation & $1313.4(440.0)$ & $1949.9(475.1)$ & $1787.3(440.0)$ & $1737.0(440.0)$ & 0.78 \\
\hline Desynchronized & $655.5(410.9)$ & $1192.9(429.2)$ & $1968.6(410.9)$ & $1006.6(410.9)$ & $<0.05$ \\
\hline \multicolumn{6}{|c|}{ Specific behaviour, frequency / $1 \mathrm{~h}$} \\
\hline Sniffing & $6.4(0.8)^{\mathrm{a}}$ & $4.4(0.9)^{\mathrm{ab}}$ & $3.4(0.8)^{b}$ & $2.0(0.8)^{b}$ & $<0.01$ \\
\hline Rubbing & $0.1(0.2)$ & $0.0(0.2)$ & $0.0(0.2)$ & $0.6(0.2)$ & 0.11 \\
\hline Trembling & $0.0(0.1)^{b}$ & $0.0(0.1)^{b}$ & $0.0(0.1)^{b}$ & $0.4(0.1)^{\mathrm{a}}$ & $<0.05$ \\
\hline Head shaking & $0.1(0.5)^{\mathrm{c}}$ & $0.6(0.5)^{\mathrm{bc}}$ & $1.8(0.5)^{\mathrm{b}}$ & $3.3(0.5)^{\mathrm{a}}$ & $<0.0001$ \\
\hline
\end{tabular}

242

243

244

245

246

247 Our hypothesis for this present study was refuted because the catheterization procedure triggered more evident short-term responses in the sows compared with the other procedures, including the

249 TUB. Non-surgical cannulation methods have been widely used for sequential blood collection in 250 clinical research in pigs to minimize stress, pain and anxiety during sampling against inserting the 251 needle multiple times. Zanella and Mendl (1992) reported that a similar technique for jugular 252 catheterization in sows did not affect salivary cortisol concentrations between 1 and $2 \mathrm{~h}$ post-

253 treatment. Moreover, other evidence also demonstrated that the identical method using the jugular

254 vein in young pigs tended to increase plasma cortisol concentrations $1 \mathrm{~h}$ post-treatment, but had no 255 effect subsequently (Carroll et al., 1999). Nevertheless, to the best of our knowledge, there are only 
256 few studies reporting on the impact of the procedure on cortisol levels in pigs. The catheterization

257 procedure in the current study was performed within $10 \mathrm{~min}$, and caused an increase in sow salivary

258 cortisol levels in the meantime. Handling or snaring sows for catheterization might not be the sole

259 reason for the effect on cortisol levels during the procedure, as those factors in the present study

260 were standardized with the treatment for the snaring group. On the other hand, for instance,

261 additional factors, such as inserting vinyl tubes or bandaging the neck of a folded ear, could

262 contribute to elevation of the salivary cortisol levels in sows during the procedure. Glucocorticoids

263 are known to take time to adjust a new situation (De Boer et al., 1988; Geverink et al., 1999). This

264 may support the finding that higher salivary cortisol levels in catheterized sows were recorded even

265 when they were returned to recover in the individual pen. However, based on the previous findings,

266 it can be assumed that the elevated salivary cortisol caused by catheterization may not last longer

267 than one hour (Carroll et al., 1999; Zanella and Mendl, 1992). Nonetheless, further studies are

268 needed to examine potential factors affecting cortisol levels during this procedure and their effects

269 in the long term period, and to establish the most suitable time for collecting consistent blood

270 samples through the cannula.

271 A previous study examined the technical and practical aspects of the TUB technique for luteal

272 tissue sampling, identifying several issues including effects of weight and age on success rate of

273 biopsy and their impacts on reproductive performance (Björkman et al., 2016). In spite of a number

274 of practical advantages from the technical aspect, the current study examined possible pain or

275 distress induced by the TUB that could represent a welfare problem. In contrast to our current

276 findings, Geverink et al. (1999) showed that a shot biopsy through a cannula to obtain a muscle

277 sample increased saliva cortisol concentrations in gilts compared with the situation before the

278 procedure, and also tended to cause a greater cortisol response in gilts compared with non-treated

279 gilts 30 min after the procedure. Moreover, in the same study, they established that more flinching

280 and rubbing behaviour occurred in gilts in the biopsy group in response to the procedure (Geverink 
281 et al., 1999). This also contrasted with our findings, showing that obvious acute pain or distress was

282 not observed in the TUB sows over the short term, and that other post-operative pain-related

283 behaviour in the TUB sows did not differ from that for non-treated sows over $1 \mathrm{~h}$ post-treatment.

284 One possible explanation could be that the genital organs, including the ovary, have no somatic

285 nerve supply and thus not sensory receptors compared to muscle tissue (König and Liebich, 2004).

286 Therefore, visceral pain could only be triggered by rapid stretching of the capsula surrounding the

287 organ, i.e. ovary, which occurred rarely during the TUB procedure (Björkman et al., 2016).

288 Furthermore, several studies demonstrated that transvaginal puncture of the ovary did not affect

289 behavioural and physiological signs of acute stress in heifers and cows (Lauffenburger et al., 1999;

290 Chastant-Maillard et al., 2003; Petyim et al., 2007). Consequently, on the basis of the current

291 findings which demonstrated no short-term treatment impact on cortisol or pain-related behaviour,

292 the results suggest that the TUB process for luteal tissue collection may not cause substantial short-

293 term pain or distress to the sow when it compared with the PAL process. In addition, the study by

294 Björkman et al. (2016) already showed that the long-term complications with the TUB procedure

295 rarely observed in sows.

296 There is no obvious explanation for the somewhat higher salivary cortisol concentrations for the

297 TUB group compared with the others before the stressor application. Previous evidence suggests

298 that salivary cortisol has a circadian rhythm that could be influenced by heredity, age, gender and

299 time of the day (Hillmann et al., 2008; Larzul et al., 2015; Ruis et al., 1997). Despite such factors as

300 breed, parity and body weight being randomly distributed, the time of sampling might have changed

301 slightly among treatments in the current experiment. All experimental sows in the research farm

302 were adapted to a fixed time for feeding at 0800 and $1600 \mathrm{~h}$. The clock time of sampling, i.e.

303 between 1200 and $1500 \mathrm{~h}$, was therefore designed to reduce this potential confounding variables, as

304 it is suggested that the expectation of feeding could affect the cortisol concentrations (Hillmann et

305 al., 2008). There might also be a risk that different sampling days according to the treatment could 
be an additional factor for the confounding consequences. However, this experimental setup,

307 conducting the PAL and TUB groups on the same day and the others on the following day, may

308 allow us to better standardize cortisol circadian patterns, since they were designed as controls and

309 treatments, respectively.

310 Sows screamed when snared to the crate, and resisted the tightening rope. However, our present

311 results showed no short-term differences in their salivary cortisol levels compared with those of the

312 non-snared sows but palpated through rectum. Furthermore, our finding that a slight increase in the

313 cortisol levels of the snaring sows after the procedure was not significant compared with the other

314 groups seems to be in agreement with previous findings (Soede et al., 2007; Merlot et al., 2011).

315 We therefore suggest that a snaring challenge might not induce a significant stress response in terms

316 of salivary cortisol status when applied in the short term. Probably our results would have been

317 clearer if the sows were not treated in any way, but we could not have separated between effects of

318 palpating and snaring. Furthermore, the stress response seen in the treatments with palpating was

319 very mild. The stress response of control sows without any treatments would not likely have been

320 different to those other groups.

321 Irrespective of handling or management systems, Strawford et al. (2008) found that there were

322 more scratches to the body in the younger sows, as they are attacked more often by the older sows

323 in group housing system, and that this could result in increasing cortisol levels in the younger sows.

324 Therefore, our findings that the cortisol concentrations increased with younger parity might be due

325 to the stress induced by being attacked by the older sows in the pen.

326 The current finding of a negative interrelationship between sniffing behaviour and salivary cortisol

327 levels could support the suggestion that reduced exploration behaviour might be associated with

328 pain in castrated pigs (Hay et al., 2003). In addition, reduction in activities is commonly associated

329 with animal in pain (Hay et al., 2003; Morton and Griffiths, 1985), and such animals were more

330 often isolated and desynchronized with their littermates (Arnold, 1985). The present study revealed 
331 that the duration of sow activities and social cohesion regarding their partner $1 \mathrm{~h}$ post-treatment did

332 not differ among treatments, with the exception of the snaring group, which showed higher

333 tendencies for sitting and desynchronizing behaviour compared with the other group. However, the

334 catheterized sows in this research often exhibited more trembling and head shaking, which could be 335 considered to indicate pain, as reported previously (Morton and Griffithis, 1985; Noonan et al.,

336 1994). Furthermore, the proven interrelationship between such pain-related behaviour and salivary

337 cortisol levels in the current study also suggests that the catheterization procedure might cause pain 338 and stress to sows during the procedure or within one hour post-treatment.

341 Present study confirmed that salivary cortisol was associated with some specific behaviour

342 response, indicating pain or distress. We found that the transvaginal ultrasound-guided biopsy of

343 luteal tissue in sows did not induce an acute pain or stress response during the procedure, but the

344 non-surgical catheterization method performed in the present study could increase salivary cortisol

345 concentrations and frequencies of pain-related behaviour in sows. Present experiment demonstrated

346 that snaring might not cause an increase in cortisol levels during the process. Nonetheless, it cannot

347 preclude the possibility that the catheterization procedure was not the sole reason for increased

348 salivary cortisol levels and frequencies of pain-related behaviour during the procedure. We might

349 also not expect that the trend towards higher cortisol levels last longer than an hour after the

350 catheterization procedure. Further studies therefore are needed to investigate the causal relationship

351 between the catheterization procedure and practical acute pain or distress, and to establish the

352 optimal time for obtaining uniform blood samples through the cannula.

354 Acknowledgments 
355 This study was funded by the Ministry of Agriculture in Finland (Dnro 2017/312/2011), and also

356 supported by a grant by Vetcare Ltd., Atria Ltd., Figen Ltd. and Agrimarket Ltd. We wish to thank 357 Jari Ollikkala (farm staff) for making his farm and animals available for this study

References

360

Arnold, G. W. (1985). Territoriality. In 'World Animal Science. Part A5: Ethology of Farm Animals'. (Ed. A.F. Fraser, pp. 249-264. (Elsevier, Amsterdam).

Björkman, S., Yun, J., Niku, M., Oliviero, C., Soede, N., Peltoniemi, O. (2016). Serial transvaginal ultrasound-guided biopsy of the porcine corpus luteum in vivo. Reprod. Fertil. Dev. http://dx.doi.org/10.1071/RD15435.

Carroll, J. A., Daniel, J. A., Keisler, D. H., and Matteri, R. L. (1999). Non-surgical catheterization of the jugular vein in young pigs. Lab. Anim. 33(2), 129-134.

Chastant-Maillard, S., Quinton, H., Lauffenburger, J., Cordonnier-Lefort, N., Richard, C., Marchal, J., Mormede, P. \& Renard, J. P. (2003). Consequences of transvaginal follicular puncture on wellbeing in cows. Reprod. 125(4), 555-563.

Conley, A. J. and Ford, S. P. (1989). Direct luteotrophic effect of oestradiol-17 beta on pig corpora lutea. J. Reprod. Fertil. 87, 125-131.

Cook, N. J., Hayne, S. M., Rioja-Lang, F. C., Schaefer, A. L., and Gonyou, H. W. (2013). The collection of multiple saliva samples from pigs and the effect on adrenocortical activity. Canadian J. Anim. Sci. 93(3), 329-333.

De Boer, S. F., Slangen, J. L., and Van der Gugten, J. (1988). Adaptation of plasma catecholamine and corticosterone responses to short-term repeated noise stress in rats. Physiol. Behav. 44(2), 273280. 
378 Geverink, N. A., Ruis, M. A., Eisen, R., Lambooij, E., Blokhuis, H. J., and Wiegant, V. M. (1999).

379 The effect of shot biopsy on behavior, salivary cortisol, and heart rate in slaughter pigs. J. Anim.

380 Sci. 77(7), 1614-1619.

381 Hänninen, L. and Pastell, M. (2009). CowLog: Open-source software for coding behaviors from 382 digital video. Behav. Res. Methods, 41(2), 472-476.

383 Hay, M. A., Vulin, A., Génin, S., Sales, P., Prunier, A. (2003). Assessment of pain induced by 384 castration in piglets: behavioral and physiological responses over the subsequent 5 days. Appl. 385 Anim. Behav. Sci. 82, 201-18.

386 Hillmann, E., Schrader, L., Mayer, C., and Gygax, L. (2008). Effects of weight, temperature and 387 behaviour on the circadian rhythm of salivary cortisol in growing pigs. Anim. 2(3), 405-409.

388 König, H. E. and Liebich, H. G., 2004. Female genital organs. Vet. Anat. of Domestic Mamm. pp. 389 397-413. (Schattauer GmbH, Germany).

390 Larzul, C., Terenina, E., Foury, A., Billon, Y., Louveau, I., Merlot, E. and Mormede, P. (2015). The 391 cortisol response to ACTH in pigs, heritability and influence of corticosteroid-binding globulin. 392 Anim. 9(12), 1929-1934.

393 Lauffenburger, J., Quinton, H., Richard, C., Marchal, J., Mormede, P., Renard, J. P., \& Chastant394 Maillard, S. (1999). Evaluation of stress associated to ovum pick-up in cow. In 15th Scientific 395 Meeting AETE (p. 178).

396 Merlot, E., Mounier, A.M., Prunier, A. (2011) Endocrine response of gilts to various common 397 stressors: a comparison of indicators and methods of analysis. Physiol. \& behave. 102(3), 259-265.

398 Morton, D. B. and Griffiths, P. H. (1985). Guidelines on the recognition of pain, distress and 399 discomfort in experimental animals and an hypothesis for assessment. Vet. Rec. 116(16), 431-436. 
401 of piglets undergoing tail docking, teeth clipping and ear notching. Appl. Anim. Behav. Sci. 39, 20340213.

403 Peacock, A.J. (1991). Environmental and social factors affecting seasonal infertility of pigs. $P h D$ -

404 thesis. University of Sydney.

405 Peltoniemi, O.A.T., Easton, B.G., Love, R.J., Klupiec, C., Evans, G. (1995). Effect of chronic 406 treatment with a GnRH agonist (Goserelin) on LH secretion and early pregnancy in gilts. Anim. 407 Reprod. Sci. 40, 121-133.

408 Petyim, S., Båge, R., Madej, A., \& Larsson, B. (2007). Ovum Pick-up in Dairy Heifers: Does it 409 Affect Animal Well-being?. Reprod. Domest. Anim. 42(6), 623-632.

410 Ribeiro, L. A., Bacci, M. L., Seren, E., Tamanini, C. and Forni, M. (2007). Characterization and 411 Differential Expression of Vascular Endothelial Growth Factor Isoforms and Receptors in swine 412 corpus luteum throughout estrous cycle. Mol. Reprod. Dev. 74, 163-171.

413 Ruis, M. A., Te Brake, J. H., Engel, B., Ekkel, E. D., Buist, W. G., Blokhuis, H. J., and Koolhaas, J. 414 M. (1997). The circadian rhythm of salivary cortisol in growing pigs: effects of age, gender, and 415 stress. Physiol. Behav. 62(3), 623-630.

416 Soede, N. M., Roelofs, J. B., Verheijen, R. J. E., Schouten, W. P. G., Hazeleger, W., \& Kemp, B. 417 (2007). Effect of repeated stress treatments during the follicular phase and early pregnancy on 418 reproductive performance of gilts. Reprod. Domest. Anim. 42(2), 135-142.

419 Strawford, M.L., Li, Y.Z., Gonyou, H.W. (2008). The effect of management strategies and parity on 420 the behaviour and physiology of gestating sows housed in an electronic sow feeding system.

421 Canadian J. Anim. Sci. 88(4) 559. 
422 Tast, A., Halli, O., Ahlstrom, S., Andersson, H., Love, R.J., Peltoniemi, O.A.T. (2001). Seasonal

423 alterations in circadian melatonin rhythms of the European wild boar and domestic gilt. J. Pineal

424 Res. 30, 43-49.

425 Virolainen, J. (2005). Post-breeding effects of feeding on reproduction in gilts and sows. Doctoral 426 dissertation, Faculty of Vet. Med., Univ. of Helsinki.

427 Weary, D. M., Niel, L., Flower, F. C., and Fraser, D. (2006). Identifying and preventing pain in 428 animals. Appl. Anim. Behav. Sci. 100(1), 64-76.

429 Yun, J., Swan, K. M., Vienola, K., Farmer, C., Oliviero, C., Peltoniemi, O., and Valros, A. (2013).

430 Nest-building in sows: effects of farrowing housing on hormonal modulation of maternal

431 characteristics. Appl. Anim. Behav. Sci. 148(1), 77-84.

432 Zanella, A. J. and Mendl, M. T. (1992). A fast and simple technique for jugular catheterization in 433 adult sows. Lab. Anim. 26(3), 211-213. 